\title{
Characterization of Waferstepper and Process related Front- to Backwafer overlay Errors in Bulk Micro Machining using Electrical Overlay Test Structures
}

\author{
H. W. van Zeijl ${ }^{* a}$, F. G. C. Bijnen ${ }^{\mathrm{b}}$ and J. Slabbekoorn ${ }^{\mathrm{a}}$ \\ ${ }^{a}$ Delft Institute of Microelectronics and Submicron technologies, Feldmannweg 17, 2628 CT Delft, \\ The Netherlands \\ ${ }^{\mathrm{b}}$ ASML Special Applications, De Run 6501, 5504 DR Veldhoven, The Netherlands
}

\begin{abstract}
To validate the Front- To Backwafer Alignment (FTBA) calibration and to investigate process related overlay errors, electrical overlay test structures are used that requires FTBA [1]. Anisotropic KOH etch through the wafer is applied to transfer the backwafer pattern to the frontwafer. Consequently, the crystal orientation introduces an overlay shift. A double exposure method is presented to separate the process-induced shift from the FTBA shift. The process induced overlay shift can run up to $3 \mu \mathrm{m}$, large compared to the expected FTBA error (around $0.1 \mu \mathrm{m}$ ). The measured overlay distribution is $0.45 \mu \mathrm{m}(3 \sigma)$, this includes both waferstepper and process related overlay errors. The overlay distribution, corrected for waferstepper related overlay errors, like lens distortion, resembles the overlay distribution of the bulk micromachining (BMM) process; $0.26 \mu \mathrm{m}(3 \sigma)$. The procedures described in this work provide a quantitative method of describing the waferstepper and process related front to backwafer overlay errors.
\end{abstract}

Keywords: Bulk Micro Machining KOH etching, Front to Backwafer Alignment, electrical overlay measurements.

\section{INTRODUCTION}

Front- To Backwafer alignment (FTBA) is a key enabling technology for the fabrication of many MEMS devices, particularly those devices that require anisotropic etching through the wafer. Waferstepper manufacturers have recognized this and developed dedicated FTBA hardware. Contrary to conventional front to frontwafer alignment, the calibration procedures for FTBA systems are more complicated. Once calibrated, a test device must be fabricated to validate the calibration procedure. The test device must be sensitive for overlay errors, and the process flow must be kept simple, preferably not exceeding two masking layers. In this work we propose the use of electrical overlay test structures in combination with KOH Bulk Micro Machining (BMM) to validate an FTBA calibration. The challenge in this approach is to separate the process induced overlay errors or shifts from a calibration offset. However when process and FTBA overlay errors can be separated, not only the calibration is checked, also the bulk micro machining process required to fabricate the device is characterized. In return, a better characterization of the process related overlay errors can lead to improved specification on materials and processes to improve the overlay performance of the BMM process.

\section{FTBA OVERLAY CALIBRATION AND MEASUREMENT}

Throughput, resolution and overlay are the most important performance indicators for wafersteppers. Especially for overlay, many different machine parameters can be adjusted to optimize the overlay performance. These parameters can be divided into two groups, intra field parameters and inter field parameters. Examples of intra field parameters are field- or die rotation, magnification and lens distortion. Important inter field parameters relate to the alignment system and the XY stage, such as alignment offsets and XY orthogonality. Generally a large number of measurements over the whole wafer are required to calibrate and optimize the overlay parameters. This is also true for wafersteppers with Front- To Backwafer Alignment (FTBA) systems, however, additional issues like substrate thickness, substrate flatness and surface parallelism can interfere with the calibration and optimization of FTBA systems.

The performance of the frontwafer alignment system of an ASML PAS 5000 waferstepper, used in this work, is

* henkz@dimes.tudelft.nl; phone +31 152784949; fax +31 152622163 
evaluated using a standard overlay test procedure. On a $100 \mathrm{~mm}$ wafer with two primary alignment markers, 9 image fields are exposed using a reticle with an array of $11 \times 11$ markers in the $15 \times 15 \mathrm{~mm}^{2}$ image field. The layout on the wafer is shown in figure 1. Using the same reticle, a second layer is exposed with a programmed shift. The wafer is developed after the exposures, revealing layer 1 and layer 2 images of the markers in the photoresist. Subsequently the wafer is loaded in the waferstepper again and the alignment system is used to measure the positions of the markers in the image fields. The difference between the measured vs. theoretical positions of corresponding markers in both layers is the overlay error.

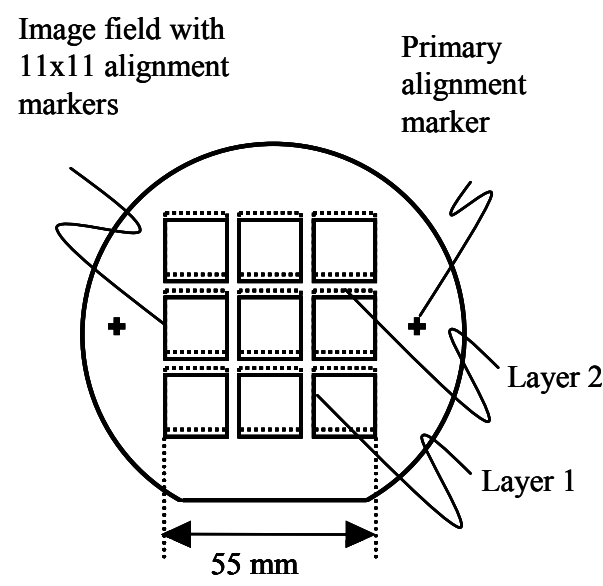

$100 \mathrm{~mm}$ wafer

Figure 1a
Frequency $(\%)$

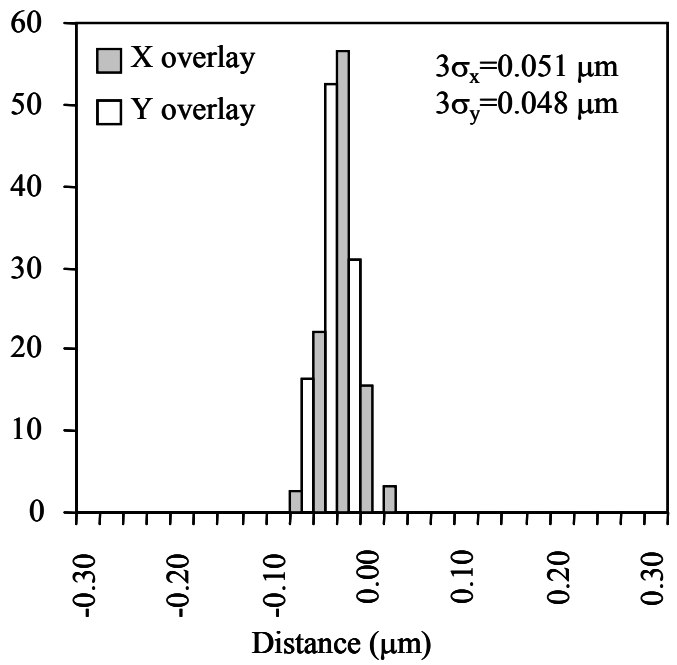

Figure 1. Field layout of the overlay test pattern (figure 1a) and measured front- to frontwafer overlay using the waferstepper alignment system as a metrology tool (figure 1b).

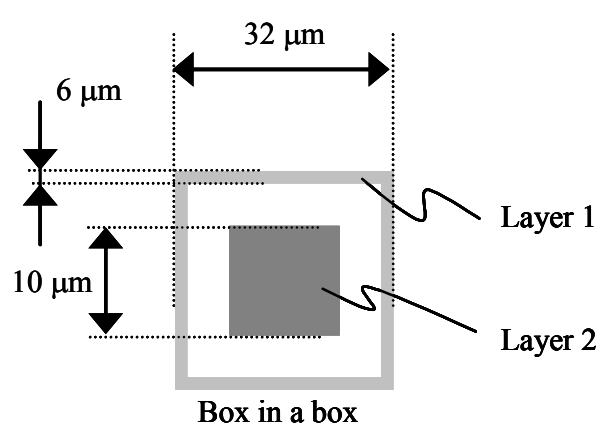

Figure 2. Typical optical overlay test structures for optical overlay measurements. calculate the back-wafer mark positions in front-wafer (exposure) coordinates and to compensate for the systematic errors introduced by the optical branches. These errors can be characterized by 4 parameters: image translation in X, translation in $\mathrm{Y}$, - rotation and - magnification. To calibrate out these errors a special silicon calibration wafer is employed with two primary flat edges opposite each other. Next to standard oriented marks, a set of $180^{\circ}$ rotated marks in a special arrangement are present on this calibration wafer. The position of these marks is determined in various wafer orientations. The effects of alignment inaccuracy and exposure offsets are reduced respectively eliminated by comparing the mark positions of all relevant orientations. This results in an over-determined set of equations characterizing both this special wafer and the FTBA optics. From these data, values for the 4 parameters mentioned above, can be derived. Once a FTBA calibration wafer has been created, calibrating other FTBA systems can be executed based on (copies of) this wafer.

In a different approach, dedicated overlay measurement tools are used, the measurement principle is based on optical pattern recognition of customized targets. Again, a large number of targets are measured over the whole wafer. A typical layout of an optical overlay target is shown in figure 2 .

Conventional frontwafer overlay characterization and calibration is impracticable if one of the corresponding layers is at the backwafer; for FTBA systems a different approach is required. The FTBA alignment system of the ASML PAS5000 consists of two sets of optics, embedded below the wafer in the XY-stage of the waferstepper [1]. The advantage of this arrangement is that the frontwafer alignment system is also used for backwafer alignment through additional optics. To relate a position on the back side of the wafer to its front wafer position, two back-wafer marks are projected from the back side to the front side of the wafer by two optical branches (see figure 3). The metrology challenge is to 


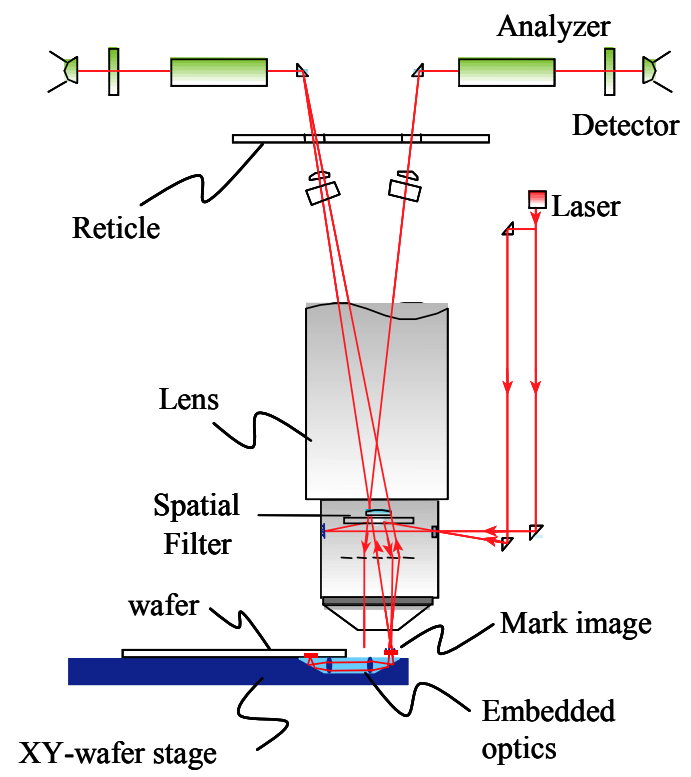

Figure 3. Overview of the FTBA alignment system of the ASML PAS5000 waferstepper.
Standard optical procedures cannot be used to verify the calibration procedure as described above, as the wafers are opaque for the alignment laser light used. Also on wafers, transparent for alignment light, overlay measurements based on pattern recognition are impractical; the limited focal depth of large magnification optics used to capture the image does not allow a sharp image of both layers of the target. Refocusing requires a large movement in the z-axis, possibly affecting calibration and stability. Dedicated Front- to Back overlay measurement tools like EVG-40 (EVGroup, Raaba, Austria) are commercially available but the reported $3 \sigma$ accuracy of $0.5 \mu \mathrm{m}$ is not accurate enough for a precise FTBA verification.

As an alternative, structures exposed on the backwafer can be transferred to the frontwafer by Bulk Micro Machining (BMM) processing. This facilitates the use of conventional overlay measurement methods on the frontwafer. The disadvantage however is that the BMM process contributes to the FTBA overlay variations. Nevertheless, a careful analysis of the overlay data can separate the contribution of the waferstepper and the BMM process. In this way, the BMM process can be characterized as well. Processes for BMM like anisotropic Deep Reactive Ion Etching (DRIE) or $\mathrm{KOH}$

etching can be used, for example to create a box in a box structure (see fig. 2) where layer 2 is exposed and etched on the backwafer. The bulk micromachining process characteristics will now influence the front- to back overlay. For example, the anisotropy and directionality of DRIE is influenced by many machine dependent parameters; thus affecting the resulting measured overlay. Anisotropic KOH etches along the (111) crystal planes, consequently, on $\mathrm{KOH}$ etched wafers the overlay is shifted by the tilt of the crystal orientation with respect to the wafer surfac. The crystal orientation is a precisely characterized material property. For that reason, $\mathrm{KOH}$ etching is preferred above DRIE. However the size

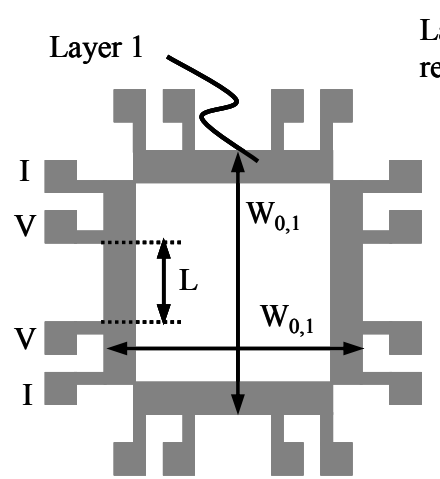

Figure $4 \mathrm{a}$
Layer 2, separating 4 resistors

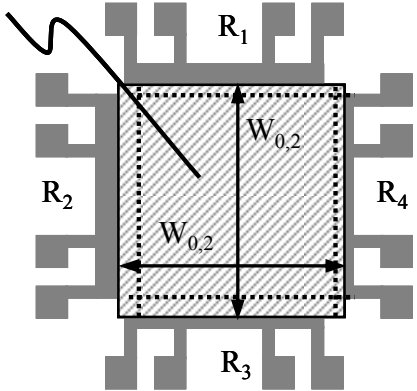

Figure $4 b$
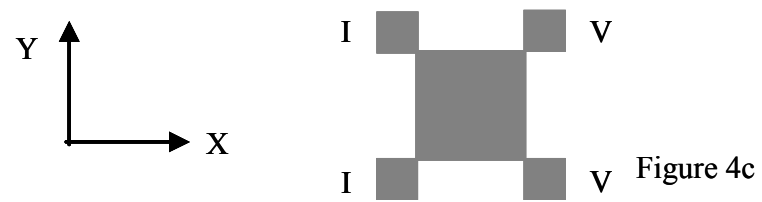

Figure 4. Electrical overlay test pattern with Kelvin probing on each resistor (figures $4 \mathrm{a}$ and $4 \mathrm{~b}$ ) and a vanderPauw structure [4] to measure the thin film sheet resistance (figure $4 c$ ).

of the box in the box (figure 2) is now dependant on the wafer thickness. Variations over the wafer and from wafer to wafer can run op to 2 to $10 \mu \mathrm{m}$ respectively. This leads to a sizing error in the $\mathrm{KOH}$ etched window of 3 to 14 micron respectively. Furthermore each 0.1 degree offset in crystal lattice orientation results in an overlay shift of 1.3 $\mu \mathrm{m}$. Evidently an overlay test structure with a large capture range is required to cope with the large sizing variations and overlay shifts associated with $\mathrm{KOH}$ etching.

The expected variations are too large to be covered by a standard optical overlay target as shown in figure 2, and as an alternative electrical overlay structures are proposed. The principal of electrical overlay measurement structures is shown in figure 4 and discussed in [1]. The test structure includes two layers sequentially patterned in the same thin conductive film. The first layer (layer 1), consists of probe pads and a set of combined resistors. The second layer (layer $2)$, defines an area in which the conductive 
material is removed in subsequent etching. Layer 2 is exposed and etched on the backwafer, the bulk micromachining etch is also used to etch the exposed resistor material as seen from the backwafer. Consequently the width of the resistors is also defined by the placement accuracy of layer 2. A current I is forced through the set of resistors and the voltage drop over each resistor is measured on separated voltage sensing leads (Kelvin probing). As described in [1], the misalignment $\left(\Delta_{\mathrm{x}, \mathrm{y}}\right)$ and layer 2 window dimensions $\left(\mathrm{W}_{\mathrm{x}, \mathrm{y}}\right)$ are calculated from the measured resistances as follows:

$$
\Delta_{\mathrm{x}}=\frac{\mathrm{L} \cdot \mathrm{R}_{\mathrm{s}}}{2} \cdot\left[\frac{1}{\mathrm{R}_{2}}-\frac{1}{\mathrm{R}_{4}}\right] \text { (1) } \quad \Delta_{\mathrm{y}}=\frac{\mathrm{L} \cdot \mathrm{R}_{\mathrm{s}}}{2} \cdot\left[\frac{1}{\mathrm{R}_{3}}-\frac{1}{\mathrm{R}_{1}}\right] \quad \text { (2) } \quad \mathrm{R}_{\mathrm{S}}=\frac{\pi}{\ln (2)} \cdot \frac{\mathrm{V}}{\mathrm{I}}
$$

where: $\mathrm{L}$ is the length of the resistors between the voltage sense points $(\mu \mathrm{m})$.

$R_{i}$ is the measured resistance of resistor $i(\Omega)$.

$\mathrm{R}_{\mathrm{s}}$ is the measured thin film sheet resistance $(\Omega)$.

$\mathrm{V}$ is the measured voltage over the vanderPauw structure $(\mathrm{V})$.

I is the current forced through the vanderPauw structure (A).

The sheet resistance can be considered as a thin film property and is measured on dedicated structures (vanderPauw structure, figure $4 \mathrm{c}$ ).

\section{ELECTRICAL OVERLAY TEST STRUCTURE; FABRICATION AND CHARACTERIZATION}

The process flow to fabricate electrical overlay test structures used to measure the FTBA performance is shown in figure 5 and described in more detail in [1]. Two alignment gratings (primary marks) are etched in a (100) silicon wafer (figure 5a), followed by the deposition of $500 \mathrm{~nm}$ LPCVD low stress silicon nitride $\left(\mathrm{SiN}_{\mathrm{x}}\right)$. A thin film of $100 \mathrm{~nm}$ PECVD TiN is then deposited on the frontwafer, exposed and etched (figure 5b); the pattern is aligned using the frontwafer alignment system on the two primary markers. Using the backwafer alignment system, layer 2 is exposed and etched in $\mathrm{KOH} 33 \%$ at $85{ }^{\circ} \mathrm{C}$ (figure 5c). The $\mathrm{SiN}_{\mathrm{x}}$ and the $\mathrm{TiN}$ are practically not etched in the $\mathrm{KOH}$ solution. The etching of layer 2 is completed using RIE from the backwafer; the wafer acts as a mask (figure 5d). To support the membranes during the last etch step, an $8 \mu \mathrm{m}$ thick spray coated photoresist film is applied before etching through the $\mathrm{SiN}_{\mathrm{x}}$ membrane.

Before each set of resistor measurements (R1, R2, R3, R4) the local sheet resistance $\mathrm{R}_{\mathrm{s}}$ is measured on a van der Pauw

Primary waferstepper frontwafer alignment marker (diffraction grating)

Figure 5a

\begin{tabular}{|ll}
\hline 100 silicon wafer & frontwafer \\
\hline
\end{tabular}

Overlay test pattern layer 1 , etched in 100 $\mathrm{nm}$ TiN

Figure 5b

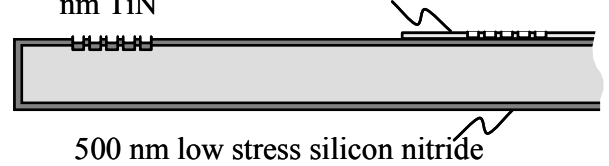

$\mathrm{KOH}$ etched cavity aligned and etched on the backwafer

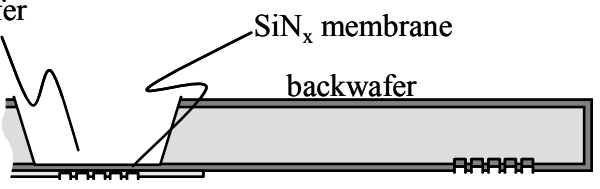

Figure 5c

plasma etched window through $\mathrm{SiN}_{\mathrm{x}}$ and $\mathrm{TiN}$

TiN electrical overlay test structure

Figure 5d

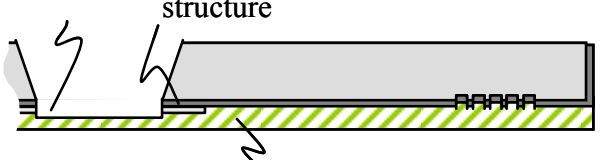

$8 \mu \mathrm{m}$ spray coated photoresist to support the membrane during $\mathrm{SiNx}$ etch and TiN etch

Figure 5. Process overview of the FTBA test device, the process steps are explained in the text.

structure [4], in this way wafer scale variations in film properties like TiN thickness and resistivity are compensated. For each overlay measurement, 5 resistor measurements are required; one sheet resistance resistor and 4 overlay resistors. The resistors are measured using a current sweep. The measured voltage drop $(\Delta \mathrm{V})$ over the resistor is a linear function of the sweep current (I) as shown in figure 6. However, the introduced measurement power $\left(\mathrm{I}^{2} \mathrm{R}\right)$ is dissipated in the 
resistor and the associated temperature variation result in a change in resistance and a non-linear V-I behavior. To avoid this ohmic heating of the resistor the sweep current is limited to $5 \mathrm{~mA}$. The repeatability of the measurement procedure is estimated as follows: the 5 resistor measurements are performed and the overlay is calculated according equation 1 and 2. This procedure is repeated 25 times on the same overlay test structure. The dispersion around the average value is shown in figure 7. Clearly the electrical measurement repeatability is sufficient for the FTBA validation.

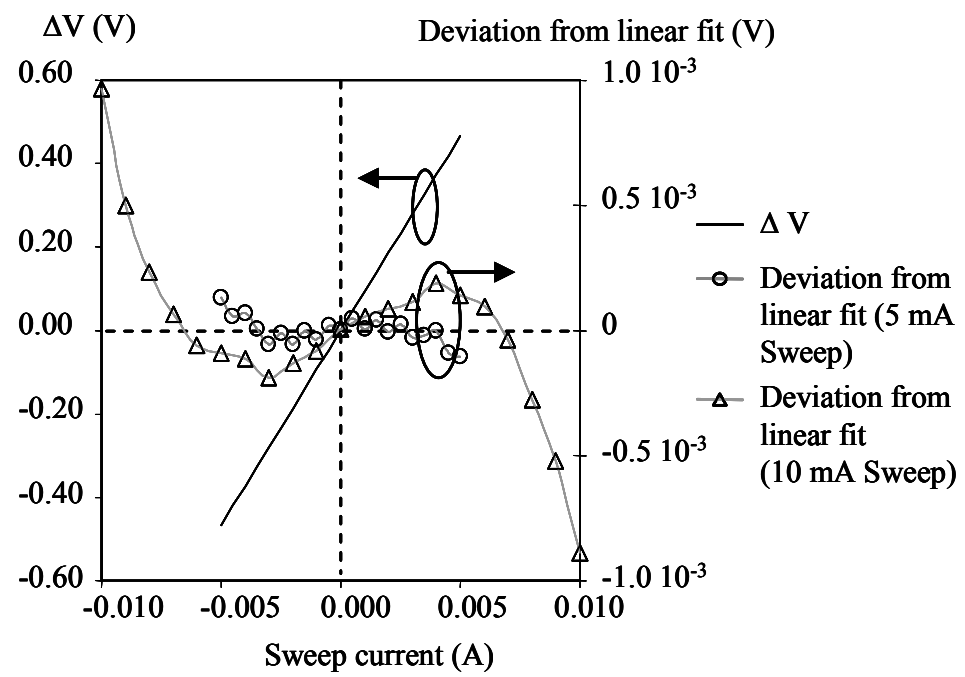

Figure 6. V-I characteristics of a measured resistor with different current sweeps.
Frequency (\%)

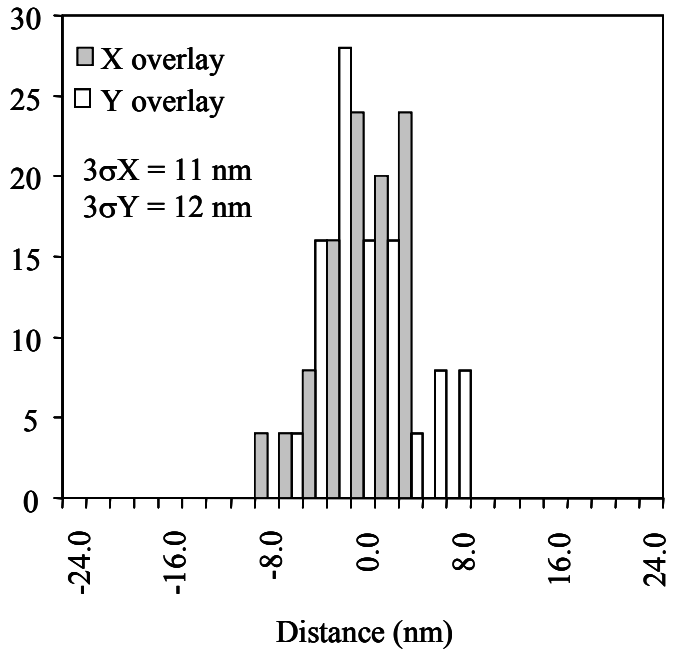

Figure 7. Dispersion of the electrical overlay reproducibility test.

The inner edges of the resistors are defined by the layer 2 etch from the backwafer. This backwafer BMM etch process completely removes the silicon over the whole wafer thickness. Usually these processes are relative violent compared with the subtle frontwafer etch process used for layer 1, in which only $100 \mathrm{~nm}$ of TiN is removed. As a result, the inner edge of the resistor may be rough or bowed which can influence the measured resistance. This can affect he electrical overlay measurements which are based on electrical linewidth measurements. The line width of a resistor ( $\left.\mathrm{W}_{R}\right)$ is calculated from the measured resistance $(R)$ and the measured sheet resistance $\left(R_{S}\right)$ as indicated in equation 4.

$$
\mathrm{W}_{\mathrm{R}}=\mathrm{R}_{\mathrm{S}} \cdot \frac{\mathrm{L}}{\mathrm{R}}
$$

where: $\mathrm{L}$ is the length of the resistors between the voltage sense points $(\mu \mathrm{m})$.

$\mathrm{R}$ is the measured resistance of resistor $(\Omega)$.

$\mathrm{R}_{\mathrm{s}}$ is the thin film sheet resistance $(\Omega)$.

It is assumed that the width is constant over the length of the resistor. However, as a result of the BMM processing, the shape of layer 2 may deviate from a perfect square. This results in resistor width variations located on the inner edge of the resistor.

In figure 8, three examples of resistor width variations are given; A tapered resistor, a resistor showing bow and one showing edge roughness. Using a Finite Element Method, the resistance of the different shapes is calculated and with equation 5 converted to an average line width. This line width is compared with the average width derived from the shape of the resistor. The difference of the geometrically derived width and the electrically derived width is the line width difference $(\Delta \mathrm{W})$ and is also shown in figure 8 . The edge roughness (RA), used in the simulations, is the arithmetic average edge roughness, calculated according to equation 5. 
$\mathrm{RA}=\frac{1}{\mathrm{n}} \sum_{\mathrm{i}}^{\mathrm{n}}\left|\Delta \mathrm{W}_{\mathrm{i}}\right|$

where: RA is the arithmetic average edge roughness $(\mu \mathrm{m})$.

$\mathrm{n}$ is the number of elements over the resistor.

$\Delta \mathrm{W}_{\mathrm{i}}$ is the difference between the average resistor width and the local resistor width at element $\mathrm{i}(\mu \mathrm{m})$.

TAPER
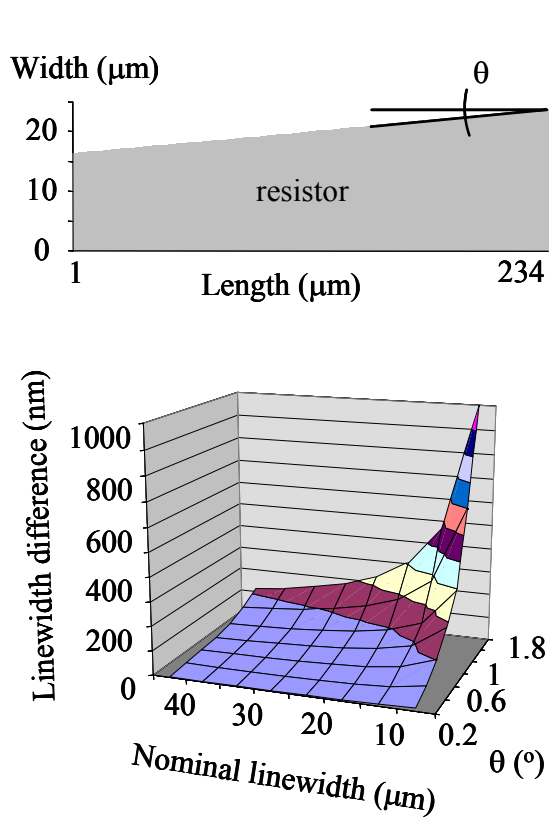

BOW
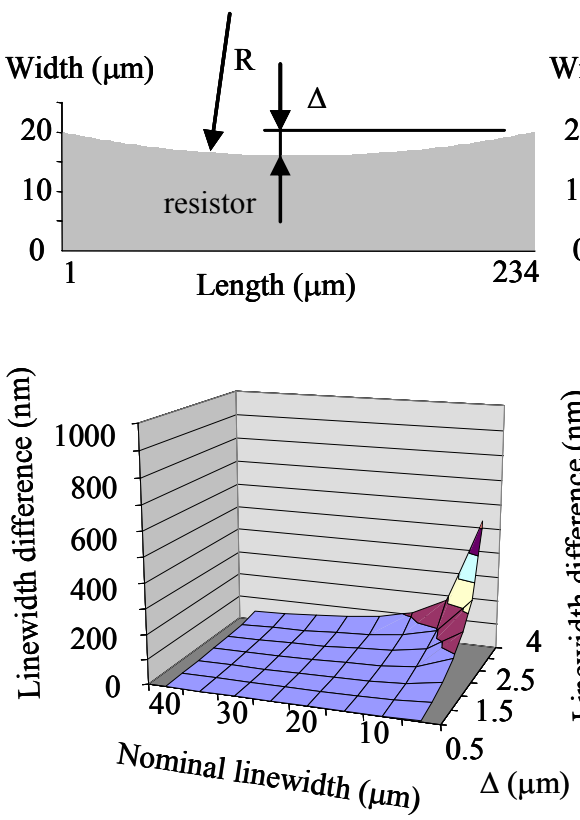

ROUGHNESS
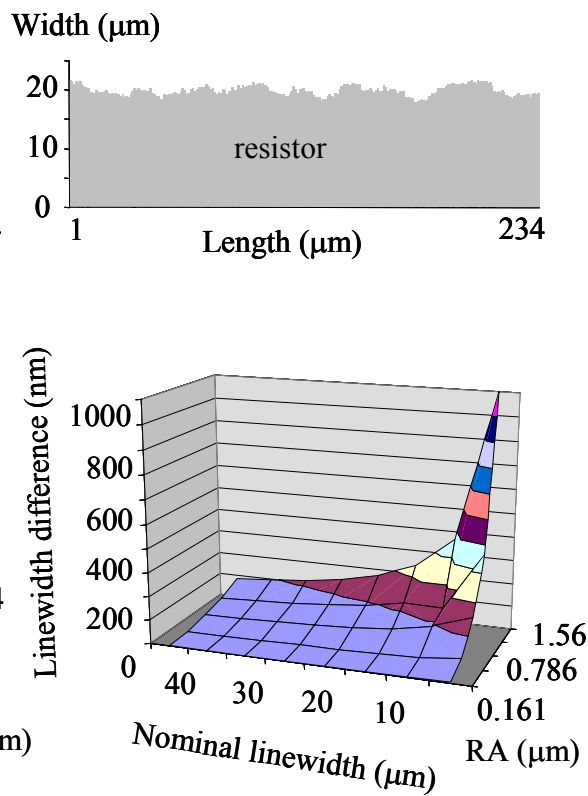

Figure 8. The influence of different resistor edge deviations on the measured line width

A maximum resistor width $\Delta \mathrm{W}$ of $1.1 \mu \mathrm{m}$ is related to a RA value of $0.16 \mu \mathrm{m}$, a $\Delta \mathrm{W}$ of $8.1 \mu \mathrm{m}$ is related to a RA value of $1.56 \mu \mathrm{m}$. Bow and edge roughness are typical deviations that can occur during DRIE. Tapered resistors shapes can be formed if $\mathrm{KOH}$ etching is used. The resistors in layer 1 are aligned orthogonal with respect to the primary flat. The $\mathrm{KOH}$ etch however follows the (111) planes. If the crystal orientation of the (111) planes is not parallel or perpendicular to the primary flat of the (100) wafer, a tapered resistor is formed after layer 2 etch.

The distortion of the resistor edge affects all resistors. This is generally the case and, consequently the line width differences will not affect the overlay measurement. However, for large overlay errors, for example $5 \mu \mathrm{m}$ in the $\mathrm{x}$ direction, the difference in resistor line width between the 2 corresponding resistors is $10 \mu \mathrm{m}$. For tapered resistors, the

\begin{tabular}{|l|l|l|l|l|l|}
\hline & \multicolumn{5}{|c|}{ Measurement error $(\mathrm{nm})$} \\
\cline { 3 - 6 } $\begin{array}{l}\text { Nominal resistor } \\
\text { width }(\mu \mathrm{m})\end{array}$ & 10 & 15 & 20 & 25 & 30 \\
\cline { 1 - 4 }$\left.\theta_{\mathrm{C}}{ }^{(}\right)$ & & & & & \\
\hline 0.5 & 47 & 17 & 9 & 6 & 4 \\
1 & 198 & 71 & 37 & 23 & 16 \\
1.5 & 492 & 71 & 37 & 23 & 16 \\
2 & 1054 & 301 & 154 & 95 & 65 \\
\hline
\end{tabular}

Table I. Overlay measurement error for an alignment offset of $5 \mu \mathrm{m}$ and different nominal resistor width and crystal angle rotations $\left(\theta_{\mathrm{C}}\right)$. measurement error is calculated for different nominal resistor widths and various off orientations $\left(\theta_{\mathrm{C}}\right)$ between the (110) plane and the primary flat edge. The crystal orientations are usually specified better than $1^{\circ}$, consequently the measurement errors are low for nominal resistor width wider than $20 \mu \mathrm{m}$. Generally the expected measurement errors for different sizing defects are low for wide resistors. The simulations can be used to tailor the resistor width to expected size offsets and alignment offsets in order to minimize the measurement error while maintaining good overlay sensitivity. 


\section{RESULTS AND DISCUSSION}

With the calibrated FTBA system two double polished $100 \mathrm{~mm}$ wafers were exposed; wafer A and wafer B. Layer 1 of the overlay test pattern is exposed on the frontwafer, layer 2, the $\mathrm{KOH}$ etch mask, is exposed on the backwafer. After the wafers are completed, the overlay is measured electrically. From the measured resistors, not only the overlay can be calculated (equations (1) and (2)), also the size of the $\mathrm{KOH}$ etched windows can be calculated using equation 6 and 7 (see [1]).

$$
\mathrm{W}_{\mathrm{x}}=\mathrm{W}_{0,1}-2 \cdot \Delta \mathrm{W}_{1}-\mathrm{L} \cdot \mathrm{R}_{\mathrm{s}} \cdot\left[\frac{1}{\mathrm{R}_{2}}+\frac{1}{\mathrm{R}_{4}}\right] \text { (6) } \quad \mathrm{W}_{\mathrm{y}}=\mathrm{W}_{0,1}-2 \cdot \Delta \mathrm{W}_{1}-\mathrm{L} \cdot \mathrm{R}_{\mathrm{s}} \cdot\left[\frac{1}{\mathrm{R}_{1}}+\frac{1}{\mathrm{R}_{3}}\right]
$$

where: $\mathrm{W}_{\mathrm{x}, \mathrm{y}}$ is the calculated window size in layer $2(\mu \mathrm{m})$.

$\mathrm{W}_{0,1}$ is the outside width of the entire resistors structure.

$\Delta \mathrm{W}_{1}$ is the sizing offset in layer 1 resulting from over etching and over exposure.

After $\mathrm{KOH}$ etching, a perfect square window must be formed. Typical lithographic pattern transfer errors are uniform in $\mathrm{x}$ and $\mathrm{y}$ direction, however the bulk micromachining process can introduce non uniform $\mathrm{CD}$ errors, for example, a pinhole in the masking nitride for $\mathrm{KOH}$ etch on the edge of the window can cause excessive under etching. This causes a sizing error of the corresponding resistor and results in a larger measured overlay vector. Since the FTBA overlay only varies in a smooth fashion over the wafer a substantial number of measurements could only be attributed to measurement errors. To reduce the influence of these errors only measurements for with $\left|\mathrm{W}_{\mathrm{X}}-\mathrm{W}_{\mathrm{Y}}\right|$ is $<1 \mu \mathrm{m}$ or below are used in the FTBA overlay calculations. For wafer A, 75\% of the data is used, for wafer B $87 \%$ of the data is used, the width of the resistors after layer 2 etch is about $30 \mu \mathrm{m}$.

The wafer layout for wafer $\mathrm{A}$ is shown in figure 9 . The front and backwafer are both exposed at rotation $0^{\circ}$ and at $180^{\circ}$. The field size is limited to minimize the contribution of lens and reticle errors to the FTBA overlay and to increase the number of dies on the wafer. These two wafer orientations allow the process-induced overlay error to be separated from the other FTBA errors in X and Y. Wet etching along the (111) planes causes the crystal orientation to contribute to a process-induced global FTBA overlay shift in $\mathrm{X}$ and $\mathrm{Y}$. This direction is determined by the wafer and is inverted for a rotation over $180^{\circ}$.

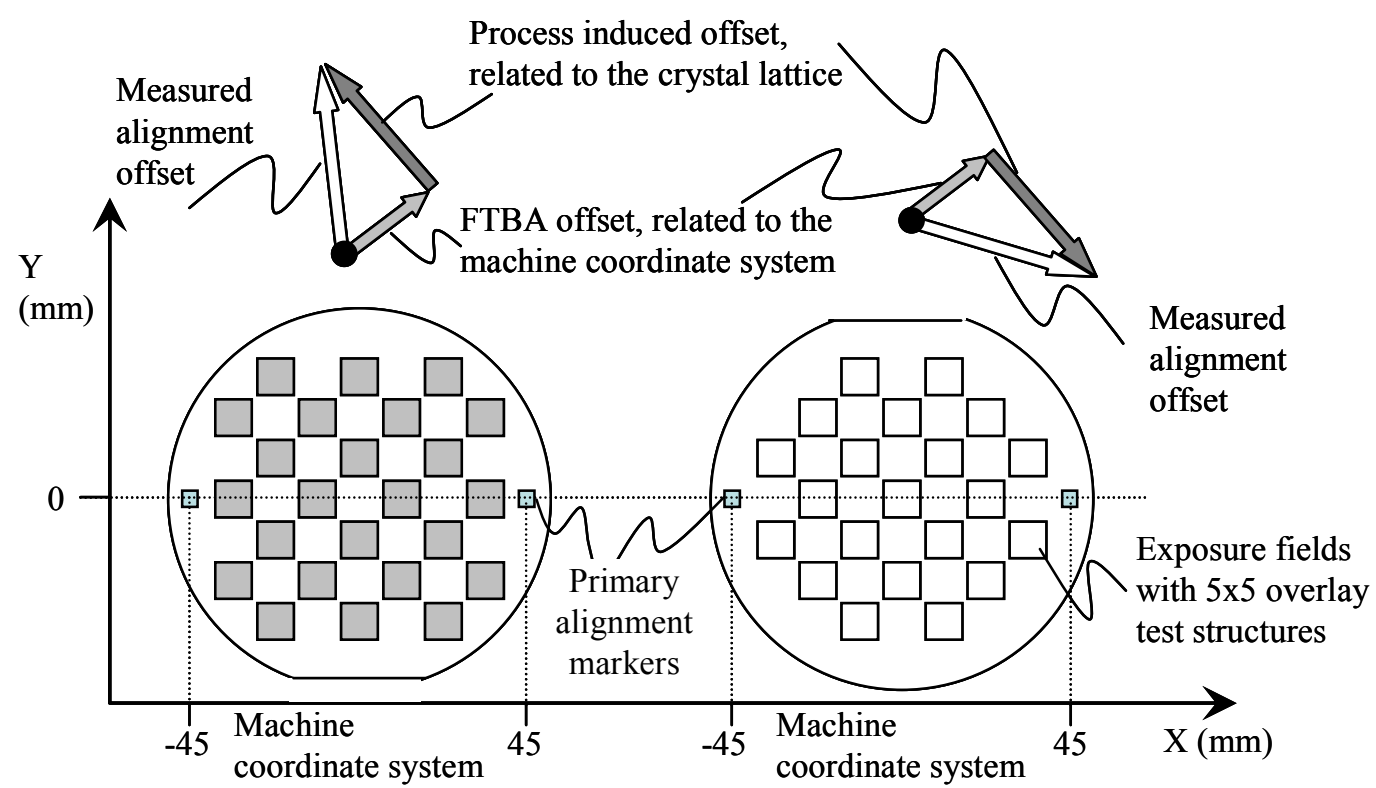

Figure 9. Two sets of exposures on the same wafer to discriminate the FTBA offset form the process induces overlay offset. 
The systematic global FTBA overlay shift in X and Y due to an error in the FTBA calibration is machine dependent. Thus the global shift in X and Y of the FTBA overlay in group I ( $0^{\circ}$ rotation $)$ and group II $\left(180^{\circ}\right.$ rotation $)$ consists of 2 sets $(\mathrm{X}$ and $\mathrm{Y})$ of 2 equations $\left(0^{\circ}\right.$ and $\left.180^{\circ}\right)$ each with 2 unknowns (process and FTBA error). See table II.

For rotation and magnification there are no quantifiable process parameters responsible. The resulting global FTBA error is for a large part due to the FTBA calibration error. The X-Y non-orthogonality of the lithographic exposure system also gives a global FTBA overlay error. This parameter is part of the standard calibration procedure of a lithographic system. This very minor contributor is basically ignored in the analysis. The $3 \sigma$ values for the averaged values of rotation, magnification, translation $\mathrm{X}$ and translation $\mathrm{Y}$ for both groups are shown in table II. The average values of the final error in table II are due to a combination of the unknown values for wafer wedge, lattice angle variations, exposure errors, alignment errors and FTBA calibration errors. Only the latter is a systematic error which can be derived more accurately by applying the electrical overlay method over more wafers.

\begin{tabular}{|l|cc|cc|lr|r|}
\hline Inter Field & Group II & $\mathrm{n}=409$ & Group II & $\mathrm{n}=413$ & Final error & FTBA error \\
parameter & Average & $3 \sigma$ & Average & $3 \sigma$ & Average & $3 \sigma$ & \\
\hline Trans X (nm) & 1492 & 30 & -1222 & 30 & 135 & 20 & $135 \mathrm{~nm}$ \\
Trans Y (nm) & -3201 & 30 & 3346 & 20 & 73 & 20 & $73 \mathrm{~nm}$ \\
Magnification $(\mathrm{ppm})$ & -5.0 & 1 & -2.5 & 1.4 & -3.8 & 1 & $-169 \mathrm{~nm}$ \\
Rotation $(\mu \mathrm{rad})$ & -1.0 & 0.8 & -0.5 & 1 & -0.8 & 0.8 & $-34 \mathrm{~nm}$ \\
\hline
\end{tabular}

Table II. The results of the Electrical overlay test. The values for groups I and II are found after filtering the results as discussed in the text. Final error value gives the result when combining the results of both Groups. For the Trans $\mathrm{X}$ and Trans $\mathrm{Y}$ values a correction for the crystal lattice effect has is thus taken into account. The $3 \sigma$ value indicates the error of the averaged values. The averages of the final error at the location of the alignment marks $( \pm 45 \mathrm{~mm})$ is given in the last column

On wafer B, 9 fields with the maximum field size $\left(15 \times 15 \mathrm{~mm}^{2}\right)$ were exposed on both sides of the wafer. Each image field contains an array of $11 \times 11$ electrical overlay test structures, similar to the standard overlay test reticle (figure 1). No rotated exposures were performed; consequently the overlay shift caused by crystal orientation cannot be calculated. However, with this wafer, the overlay data can be compared with the conventional front to frontwafer overlay since field and wafer layout is similar. The electrically measured front to backwafer overlay is shown in figure 10. Compared with conventional frontwafer lithography (figure 1b), the overlay distribution is considerably increased.This increased overlay distribution is not only caused by the BMM process. In front to backwafer lithography, the backwafer is the mirrored image of the frontwafer. Consequently the reticle, lens and stage parameters are mirrored and the overlay

Frequency $(\%)$

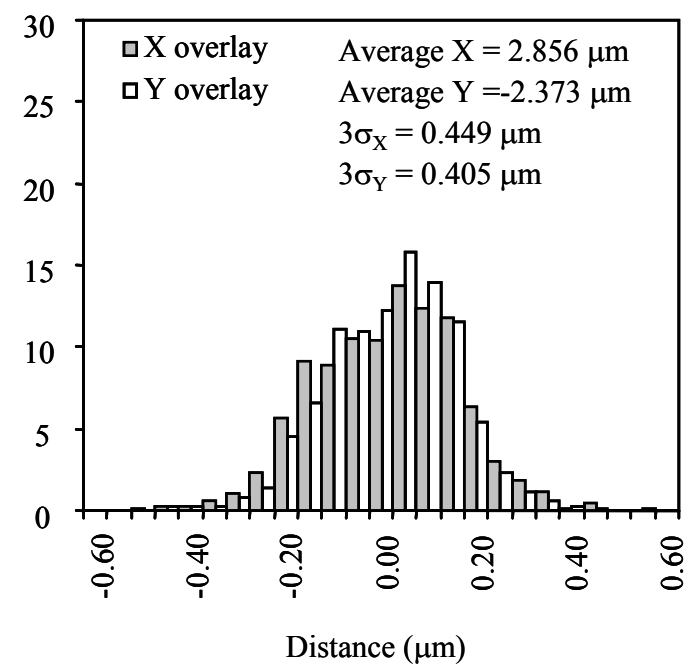

Figure 10. Electrically measured front to backwafer overlay, including reticle and lens errors. accuracy is reduced by non-mirror symmetric errors.

To estimate the contribution of the BMM process, the following procedure is followed; the intra field parameters magnification and rotation for the exposures on wafer $\mathrm{B}$ are $1.2 \mathrm{ppm}$ and $1.3 \mu \mathrm{rad}$ respectively. The data is corrected for the found wafer magnification and wafer rotation. This means that in an ideal exposure situation a specific position in a field has for all field positions a fixed Front to backside distance. The averaged sum of all standard deviations of the (electrically) measured overlay of each field position over all exposed fields yields the BMM sigma; $3 \sigma_{X}=0.263 \mu \mathrm{m}$ and $3 \sigma_{\mathrm{Y}}=0.257 \mu \mathrm{m}$. 


\section{CONCLUSIONS}

An electrical overlay test structure is successfully used to validate the calibration of a Front To Backwafer Alignment (FTBA) system. A KOH etch Bulk Micro Machining (BMM) process is used to transfer the backwafer pattern to the frontwafer. The consequence of this approach is that not only the FTBA system but also the BMM process contributes to the FTBA overlay errors. Using exposures at two orientations on both the front side and the back side of a wafer, the systematic overlay offset of the $\mathrm{KOH}$ etched BMM process is separated from an FTBA offset. The FTBA error is measured with an accuracy of approx. $0.1 \mathrm{um}$. The FTBA calibration error is included in the FTBA error. Taking into account the error of the electrical overlay method and understanding that not yet all error sources have been included the expected FTBA calibration error is in correspondence with the found FTBA error.

Simulations of the resistors with different shapes indicates that the electrical overlay test structure is not very sensitive for BMM etch related sizing errors like taper, bow and roughness. Therefore, the application of the electrical overlay test structure is not limited to $\mathrm{KOH}$ etching, but also for other BMM technologies like Deep Reactive Ion etching, where bow and roughness of the etched sidewalls can occur.

Using the potentials of advanced FTBA lithography, the materials and processes used in device fabrication may now become the limiting factor in the FTBA overlay. However, a better characterization of the process related overlay errors can lead to improved specification on materials and processes to improve the overlay performance of the BMM process.

\section{ACKNOWLEDGEMENTS}

The authors wish to acknowledge W. van Buel from ASML for his advice and assistance in the data processing and E. Smeets from ASML for carefully reading the manuscript.

\section{REFERENCES}

[1] H.W. van Zeijl, J. "Slabbekoorn, Characterization of Front- to Backwafer Alignment and Bulk Micromachining Using Electrical Overlay Test Structures", Smart Sensors, Actuators and MEMS, Proc. SPIE, Vol. 5116, May 2003, pp. 617-626.

[2]: C. Gui, W. v. Buel, F. Bijnen and J. Lof, "Extended front-to-back alignment capability for MEMS/MOEMS

Applications", Emerging Lithographic Technologies VI, Proc. SPIE Vol. 4688, 2002, pp 867-873

[3] F. Bijnen, W. v. Buel, C. Gui and J. Lof, "Front-to-back alignment metrology", Emerging Lithographic

Technologies VII, Proc. SPIE Vol. 5037, pp 641-646

[4] L.J. van der Pauw, "A method of measuring specific resistivity and hall effects of discs of arbitrary shape", Philips

Res. Rep. vol 13. pp 1-9 1958 